\title{
Emerging Technologies for Hemodialysis Access Management: Are They Worth It?
}

\author{
Karim Valji ${ }^{1}$ \\ ${ }^{1}$ Department of Radiology, University of Washington, Seattle, \\ Washington, United States \\ J Clin Interv Radiol ISVIR 2019;3:72-73
}

Almost 40 years after the first published reports, percutaneous balloon angioplasty (PTA) remains the fundamental endovascular means for restoring patency of dialysis fistula and grafts. ${ }^{1}$ Evolution of technology, such as the development of very high-pressure balloons and cutting balloons, has improved overall technical success of the procedure. Still, long-term patency remains modest. The U.S. National Kidney Foundation Dialysis Outcomes Quality Initiative (K-DOQI) guidelines set a target of 6-month primary patency after balloon angioplasty in the venous outflow of dialysis grafts of only about 40 to $50 \%{ }^{2}$ And once angioplasty has been done, the stenotic process is accelerated in many patients, leading to recurrent obstruction and sometimes complete thrombosis in a short period of time. Despite decades of research into the mechanisms underlying restenosis and potential means to combat the problem (whether systemic or directed therapy at the treatment site), significant advances have remained elusive. However, two devices that have emerged in the last decade are worthy of careful consideration by interventional radiologists (IRs).

Drug-coated balloons (DCB) are catheters with an antiproliferative agent (such as paclitaxel) impregnated on a matrix on the balloon surface. Prolonged inflation allows delivery of the agent into the vascular wall; the drug is intended to inhibit the process of restenosis at the target site. In several large clinical trials, DCB have been highly effective in reducing the rate of restenosis after balloon angioplasty of femoropopliteal arteries. ${ }^{3}$ However, these encouraging results cannot be simply extrapolated to dialysis access. Several small uncontrolled studies using paclitaxel-coated balloons showed some clinical promise. However, the results of single-center randomized trials between paclitaxel DCB and conventional PTA in patients with dysfunction dialysis fistulas were mixed. ${ }^{4,5}$

The pivotal study of DCB in dialysis access is the large multicenter trial of Trerotola and coinvestigators. ${ }^{6}$ This extremely well-designed and well-executed study enrolled 285 patients with mature but dysfunctional dialysis fistula. Patients with immature fistula, central venous stenosis, or synthetic graft-related stenosis were excluded. Notably, the primary efficacy endpoint at 6 months (lack of need for clinically mandated reintervention at the treatment site or access thrombosis) favoring DCB was not met. Nor was there any benefit in overall access circuit patency at 6 months between the two treatment arms. Of note, all patients were treated first with high-pressure angioplasty balloons to ensure adequate technical success. The authors highlighted an exploratory post hoc analysis that identified significant difference in primary patency at 210 days. Nonetheless, at this moment, given the significant added cost of DCB compared with standard high-pressure balloons (INR 42,000-100,000 vs. INR 12,50029,000 , Dr. Shyam Kumar N. Keshava, personal communication, 2019), it is premature to endorse routine use of DCB. The device may be valuable in patients with very aggressive and rapid restenosis who return within 1 to 2 months of treatment with access dysfunction over and over again, especially if they are close to exhausting possible access sites.

Since the very inception of both bare and covered stents, IRs have hoped that these devices would be the panacea for access dysfunction. The initial enthusiasm turned to disappointment as the various problems with bare metal stents in this setting were discovered. However, the potential value of covered stents has been much more encouraging. Several reports over the past 10 years are particularly notable.

The multicenter randomized RENOVA trial of Haskal and coinvestigators studied the relative value of the FLAIR stent graft versus standard PTA in patients with patent dialysis grafts and significant venous anastomotic stenosis. ${ }^{78} \mathrm{~A}$ total of 271 patients were enrolled in the study and $191 \mathrm{com}-$ pleted it. Primary target site patency (along with several other measures) was significantly better in the stent graft group than the angioplasty group at 12 months (47.6 vs. $24.8 \%$ ). Vesely et al. also found benefit for covered stents in 293 patients with synthetic dialysis grafts, $44 \%$ of whom had complete graft thrombosis. ${ }^{9}$ The AVeNEW trial has enrolled 280 patients with native dialysis fistula vein stenosis at
C2019 by Indian Society of Vascular and Interventional Radiology

\section{License terms}

$\circledast(1) \Theta \circledast$
Dol https://doi.org/ 10.1055/s-0039-1693544 Radiology, University of Washington, ISSN 2457-0214. Box 357115, 1959 NE Pacific Street, Seattle, WA 98195, United States (e-mail: kvalji@uw.edu). 
24 centers around the world. ${ }^{10,11}$ Subjects were randomized to angioplasty or the Covera covered stent graft. Superiority of the Covera device with respect to 6-month primary patency (78.7 vs. $47.9 \%$ ) was established. Unlike several prior studies that only evaluated covered stents in the cephalic arch or central veins of dialysis fistula, $40 \%$ of sites in the AVeNEW trial involved more peripheral cephalic or basilic veins, including swing point sites. Subgroup analysis suggested that the Covera stent graft may be superior to angioplasty alone at these specific locations as well (B. Dolmatch, presented at CIRSE annual meeting, September 2018).

Covered stent devices are expensive relative to standard high-pressure balloons (INR 66,000-100,000 vs. INR 12,50029,000 , Dr. Shyam Kumar N. Keshava, personal communication, 2019). Where the cost-benefit analysis falls will depend on many factors that will vary in different parts of the world. At this time, many IRs continue to rely on balloon angioplasty as first-line therapy and reserve covered stent placement for patients in whom the treatment site is amenable to device insertion and who have immediate technical failure from angioplasty alone or particularly frequent recurrent restenosis or access thrombosis.

Unfortunately, there has been a tendency over many decades for IRs to prematurely adopt new technology well before its value in patients has been proven. By that one does not mean the ability to produce a beautiful and gratifying angiogram of a newly recanalized blood vessel, but rather the clinical benefit experienced by the patient in terms of quality and length of life. Sometimes, our excitement about new devices or new versions of old ones (perhaps introduced by biased salespeople, a page on the internet or social media, or a very preliminary technical report) clouds our decision-making. The "cool case" shared on Twitter or shown in an "Extreme IR" session is lauded; some look askance at the cautious IR who waits for strong proof of safety and benefit. But we must remember: we are not really here to fix thingswe are here to help patients. And sometimes that means saying "no" (for now) to the "latest and greatest" things.

\section{Financial Disclosure}

The author has no potential conflicts of interest regarding the material presented in this manuscript.

\section{References}

1 Novelline RA. Percutaneous transluminal angioplasty: newer applications. AJR. Am J Roentgenol 1980;135(5):983-988

2 National Kidney Foundation, Vascular access. Available at: https://www.kidney.org/professionals/guidelines/guidelines_ commentaries/vascular-access. Accessed June 29, 2019

3 Rosenfield K, Jaff MR, White CJ, et al; LEVANT 2 Investigators. Trial of a paclitaxel-coated balloon for femoropopliteal artery disease. N Engl J Med 2015;373(2):145-153

4 Kitrou PM, Spiliopoulos S, Katsanos K, Papachristou E, Siablis D, Karnabatidis D. Paclitaxel-coated versus plain balloon angioplasty for dysfunctional arteriovenous fistulae: one-year results of a prospective randomized controlled trial. J Vasc Interv Radiol 2015;26(3):348-354

5 Björkman P, Weselius EM, Kokkonen T, Rauta V, Albäck A, Venermo M. Drug-coated versus plain balloon angioplasty in arteriovenous fistulas: a randomized, controlled study with 1-year follow-up (the Drecorest Ii-study) Scand J Surg 2019;108(1):61-66

6 Trerotola SO, Lawson J, Roy-Chaudhury P, Saad TF; Lutonix AV Clinical Trial Investigators. Drug coated balloon angioplasty in failing av fistulas: a randomized controlled trial. Clin J Am Soc Nephrol 2018;13(8):1215-1224

7 Haskal ZJ, Trerotola S, Dolmatch B, et al. Stent graft versus balloon angioplasty for failing dialysis-access grafts. N Engl J Med 2010;362(6):494-503

8 Haskal ZJ, Saad TF, Hoggard JG, et al. Prospective, randomized, concurrently-controlled study of a stent graft versus balloon angioplasty for treatment of arteriovenous access graft stenosis: 2-year results of the RENOVA study. J Vasc Interv Radiol 2016;27(8):1105-1114

9 Vesely T, DaVanzo W, Behrend T, Dwyer A, Aruny J. Balloon angioplasty versus Viabahn stent graft for treatment of failing or thrombosed prosthetic hemodialysis grafts. J Vasc Surg 2016;64(5):1400-1410

10 Interventional News, Covered stent provides greater patency than angioplasty alone in AV fistulae. Available at: https:// interventionalnews.com/covered-stent-angioplasty Accessed June 29, 2019

11 LINC, Prospective, multi-center study of an arteriovenous (AV) covered stent to treat stenoses at the venous anastomosis of AV graft access circuits. Available at: https://linc2018. cncptdlx.com/media/1730_Bart_Dolmatch_30_01_2018_ Room_2_-_Main_Arena_2_v1.pdf. Accessed June 29, 2019 